Pacific Journal of Mathematics

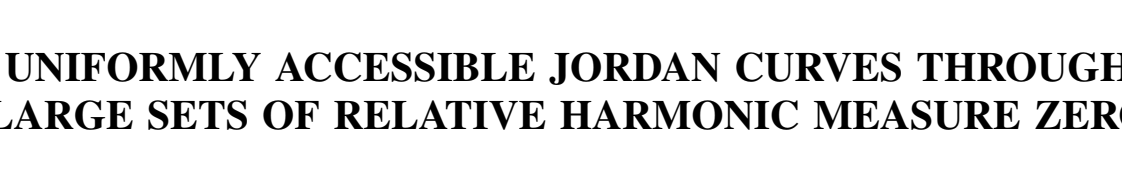




\title{
UNIFORMLY ACCESSIBLE JORDAN CURVES THROUGH LARGE SETS OF RELATIVE HARMONIC MEASURE ZERO
}

\author{
George Piranian
}

1. Introduction. Let $R$ be a Jordan region in the $w$-plane, $E$ a set of points on the boundary $C$ of $R$, and $w=f(z)$ a continuous schlicht mapping of the closed unit disc in the $z$-plane upon the closure of $R$, conformal in the open unit disc. The set $E$ is said to have harmonic measure zero relative to $R$ provided its image $f^{-1}(E)$ on the unit circle is a set of Lebesgue measure zero.

Lohwater and Seidel [4] have constructed a Jordan region $R$ whose boundary passes through a linear set $E$ of positive measure in such a way that $E$ has harmonic measure zero relative to $R$. Also, Lohwater and Piranian [3] have described a Jordan region $R$ whose boundary passes through a set $E$ of positive two-dimensional Lebesgue measure in such a way that $E$ has harmonic measure zero relative to $R$. In both cases, the set $E$ consists of points each of which is not the end point of any rectifiable arc whose remaining points lie in $R$. And in both cases the fact that the points of $E$ are not accessible by rectifiable paths in $R$ serves no other purpose than to permit the application of Lavrentiev's theorem on finite accessibility [1] (see also Tsuji [9, p. 99] and Seidel and Walsh [8, p. 143]) in proving that the set $E$ has harmonic measure zero relative to $R$.

The author of the present note holds the view that bizarre examples are acceptable only as long as no simple replacements are available; and he believes that the geometrically important aspect of the earlier constructions is not the fact that the points of $E$ are not finitely accessible from $R$, but merely this, that the points of $E$ cannot be reached from the interior of $R$ except along paths through narrow corridors (whose tortuous character is irrelevant, except in the proofs). The motivation of this belief is evident from the proof that will be given of the following proposition.

THEOREM 1. There exists a Jordan region $R$ whose boundary $C$ passes through a linear set of positive Lebesgue measure and of harmonic measure zero relative to $R$, and which has the property that each point of $C$ can be reached from a certain fixed point in $R$ by an arc whose length is less than one and whose interior points all lie in $R$.

Received January 3, 1952.

Pacific J. Math. 2 (1952), $371-375$ 
In $\$ 2$, this theorem is proved by means of the principle of the maximum. In $\S 3$, a stronger theorem is proved with the aid of Lavrentiev's theorem on angular accessibility. The last section proves a theorem concerning Jordan curves passing through sets of positive two-dimensional measure, and presents a conjecture suggested by the proof of the theorem.

2. Proof of Theorem 1. Let $L$ be the line segment joining the points $w=$ $\pm 1 / 2+i$, and let $P_{11}$ denote its midpoint. Let an open segment of length $1 / 4$ and with its midpoint at $P_{11}$ be deleted from $L$, and let $P_{21}$ and $P_{22}$ be the midpoints of the two surviving segments, with $P_{21}$ to the left of $P_{22}$. Let open segments of lengths $1 / 16$ and with their midpoints at $P_{21}$ and $P_{22}$ be deleted from what remains of $L$, and let $P_{3 r}(r=1,2,3,4)$ denote the midpoints of the surviving segments, with $P_{3 r}$ to the left of $P_{3, r+1}$. Let segments of lengths $1 / 64$ and with their midpoints at $P_{3 r}(r=1,2,3,4)$ be deleted from what remains of $L$. Let this process of deletion be continued indefinitely, and let $E$ denote the Cantor set on $L$ which survives the process. Clearly $E$ has Lebesgue measure $1 / 2$.

For $n=1,2, \cdots$, and $r=1,2, \cdots, 2^{n-1}$, let $p_{n r}$ be the projection of the point $P_{n r}$ upon the line $v=1-2^{-n}$, where $v$ denotes the imaginary part of $w$. Let $R_{1}$ consist of a (closed) narrow rectangle $Q_{11}$ in the $w$-plane, with its sides parallel to the coordinate axes, and with its short sides passing through the points $w=0$ and $w=p_{11}=i / 2$, respectively. Let $R_{2}$ consist of $R_{1}$ together with two disjoint narrow parallelograms $Q_{21}$ and $Q_{22}$, where $Q_{21}$ has one of its short sides on the upper side of $Q_{11}$ while its other short side passes through $p_{21}$; and where $Q_{22}$ has one of its short sides on the upper side of $Q_{11}$ while its other short side passes through $p_{22}$ (that is, let $R_{2}$ be in the shape of the letter $Y$, with its base at the origin, its fork at $p_{11}$, and its upper ends at $p_{21}$ and $p_{22}$ ). Let $R_{3}$ consist of $R_{2}$ together with four narrow parallelograms $Q_{3}(r=1,2,3,4)$ of which the first two reach from the free end of $Q_{21}$ to $p_{31}$ and $p_{32}$, respectively, while the other two reach from the free end of $Q_{22}$ to $p_{33}$ and $p_{34}$, respectively. Let $R$ denote the interior of the set which is obtained as this construction is continued indefinitely. Then $R$ is a Jordan region in the shape of a tree whose trunk undergoes dichotomous branching near $p_{11}$, whose branches split again near $p_{21}$ and $p_{22}$, and so on. The boundary $C$ of $R$ meets the line-segment $L$ in the set $E$. And each point of $C$ can be reached from the point $w=2 i / 5$ by a curve in $R$ of length less than 1 .

It remains only to make certain that the harmonic measure of the set $E$ relative to the region $R$ is zero. Now let $w=f(z)$ map the unit disc conformally upon $R$, with $f(0)=i / 4$. For $n=2,3, \cdots$, let $C_{n}$ denote that part of the boundary of $R$ which lies above the line $v=1-2^{1-n}$. Then, by the principle of 
the maximum, the measure of the inverse image on $|z|=1$ of the set $C_{n}$ can be made arbitrarily near to zero simply by choosing the parallelograms $Q_{n r}$ sufficiently narrow (see [6], p. 63). This completes the proof of the theorem.

3. On star-shaped Jordan regions. The Jordan region constructed by Lohwater and Seidel has boundary points that are not accessible from the interior by rectifiable arcs. The boundary points of the region constructed in the preceding section are not only finitely accessible from the interior, but they are uniformly finitely accessible. However, the new region still leaves something to be desired, because not all of its boundary points are linearly accessible from the interior.

THEOREM 2. There exists a star-shaped Jordan region $R$ whose boundary meets the circle $|w|=1$ in a set of positive Lebesgue measure and of harmonic meas ure zero relative to $R$.

Let $R_{0}$ denote the disc $|w|<1$, and let $R_{1}$ be obtained from $R_{0}$ by the deletion of an isosceles "triangle" whose boundary consists of an arc of the circle $|w|=1$ and of two rectilinear segments of length $1 / 2$. Let $R_{2}$ be obtained from $R_{1}$ by the deletion of finitely many further such isosceles triangles with one side on the circle $|w|=1$ and with rectilinear sides of length $1 / 4$. Let $R$ be obtained by the continuation of this construction, and let appropriate precautions be taken so that the boundary of $R$ meets the circle $|w|=1$ in a set $E$ of positive Lebesgue measure, and so that no point of $E$ is the vertex of an ordinary triangle whose interior points all lie in $R$. Because $R$ is star-shaped, it follows from Lavrentiev's theorem on angular accessibility [2, see part 1 of Theorem 1 on p. 822] that $E$ has harmonic measure zero relative to $R$, and the proof is complete.

The construction that has just been carried out was suggested by a region of Lusin [5]. However, the latter region is not star-shaped, as the reader will discover if he passes from the examination of Lusin's figure on p.153 to the perusal of the accompanying text.

4. The two-dimensional analogue of Theorem 1 . The method used in proving Theorem 1 can be used to extablish the following proposition.

THEOREM 3. There exists a Jordan region $R$ whose boundary $C$ passes through a set of positive two-dimensional Lebesgue measure and of harmonic measure zero relative to $R$, and which has the property that each point of $C$ can be reached from a certain fixed point in $R$ by an arc whose length is less than one and whose interior points all lie in $R$. 
Let $R_{0}$ denote the open square with vertices $w=0,1 / 2, i / 2$, and $(1+i) / 2$. Let $R_{1}$ denote the set of points $x+i y$ in $R_{0}$ which satisfy one of the two conditions $0<x<p, 0<y<p$, where $p$ is some small positive constant $(p=1 / 10$, for example). The region $R$ shall consist of $R_{1}$ together with a certain set of narrow strips whose closure nearly fills the remainder of the square $R_{0}$. For the sake of a simple description, the language used by Osgood [7] in his construction of a Jordan curve of positive area will be applied. The region $R_{1}$ is to be thought of as constituting a body of water. A narrow channel $R_{2}$ from the horizontal leg of $R_{1}$ to the upper boundary of $R_{0}$ bisects the part of $R_{0}$ not occupied by $R_{1}$. To the left of $R_{2}$ and immediately adjacent to it a dike $Q_{2}$ is erected, of equal width and length with $R_{2}$. The portion of $R_{0}$ which has not yet been used consists now of two rectangles. One of these is bisected by a horizontal channel $R_{3}$ extending from $R_{1}$ as far as $Q_{2}$; the other is bisected by a horizontal channel $R_{4}$ extending from $R_{2}$ to the right-hand edge of $R_{0}$. Parallel to each of these two channels, and immediately adjacent to it from below, a dike is erected.

The portion of $R_{0}$ which has not yet been used consists now of four rectangles; each of these rectangles is bordered by channels on the left and below, and by dikes (or edges of $R_{0}$ ) on the right and above. The cooperative reader recognizes the spirit in which the construction of channels and dikes is to be continued. The union $R$ of all the channels constructed in the process constitutes a Jordan region. All points lying on dikes are exterior to $R$, and all the “swampy" points of $R_{0}$ which lie neither in channels nor on dikes are boundary points of $R$. If at each stage of the construction the new channels and dikes are made narrow enough, the boundary $C$ of $R$ passes through a set of positive twodimensional Lebesgue measure.

Also, the principle of the maximum implies that if the channels are sufficiently narrow, then under every conformal mapping $w=f(z)$ of the unit disc upon the Jordan region $R$, a set of measure $2 \pi$ on $|z|=1$ is mapped into a set $E$ on $C$ which lies on a certain denumerable collection of rectilinear segments, that is, which has two-dimensional measure zero. It follows that a set of measure zero on $|z|=1$ is mapped upon a set of positive two-dimensional measure. Since $C$ is obviously uniformly accessible from the interior of $R$, the proof is complete.

In the case of the Jordan region $R$ constructed for the proof of Theorem 3, a set of two-dimensional measure zero on the boundary $C$ is the image of a set of one-dimensional measure $2 \pi$ on the circle $|z|=1$. This state of affairs generates a disquieting suspicion: it may be that Theorem 3 is in a certain sense a trivial consequence of the fact that some Jordan curves have positive twodimensional measure zero. Of many modes of misbehavior of functions on the 
boundaries of their domains of existence, it is true that all of the misbehavior necessarily takes place exclusively on a small set. In the light of what is already known, the following proposition appears to be reasonable:

Conjecture. Let

$$
f(z)=\sum_{n=0}^{\infty} a_{n} z^{n} \text {, with } \sum_{n=0}^{\infty} n\left|a_{n}\right|^{2}<\infty,
$$

and let $E$ be the set of points $e^{i \theta}$ for which

$$
f\left(e^{i \theta}\right)=\lim _{r \rightarrow 1} f\left(r e^{i \theta}\right)
$$

exists. Then $E$ has a subset $E^{*}$ of measure $2 \pi$ and with the property that the set of values $\left\{f\left(e^{i \theta}\right)\right\}\left(e^{i \theta}\right.$ on $\left.E^{*}\right)$ has two-dimensional Lebesgue measure zero.

\section{REFERENCES}

1. M. Lavrentiev, Sur la correspondance entre les frontiéres dans la représentation conforme, Mat. Sbornik 36 (1929), 112-115.

2. - Sur quelques propriétés des fonctions univalentes, Mat. Sbornik N. S. 1 (1936), 815-846.

3. A. J. Lohwater and G. Piranian, Conformal mapping of a Jordan region whose boundary has positive two-dimensional measure, Michigan Math. J. 1 (1952), 1-4.

4. A. J. Lohwater and W. Seidel, An example in conformal mapping, Duke Math. J. 15 (1948), 137-143.

5. N. Lusin, Sur une propriété des fonctions a carré sommable, Bull. Calcutta Math. Soc. 20 (1928), 139-154.

6. R. Nevanlinna, Eindeutige analytische Funktionen, Berlin, 1936.

7. W. F. Osgood, A Jordan curve of positive area, Trans. Amer. Math. Soc. 4 (1903), 107-112.

8. W. Seidel and J. L. Walsh, On the derivatives of functions analytic in the unit circle and their radii of univalence and of p-valence, Trans. Amer. Math. Soc. 52 (1942) 128-216.

9. M. Tsuji, On the theorems of Carathéodory and Lindelöf in the theory of conformal representation, Jap. J. Math. 7 (1930), 91-99.

The University of Michigan 



\title{
PACIFIC JOURNAL OF MATHEMATICS
}

\section{EDITORS}

\section{R. M. RobINSON \\ University of California \\ Berkeley 4, California}

\author{
*R. P. Dilwor TH \\ California Institute of Technology \\ Pasadena 4, California
}

\author{
E. F. BECKENBACH, Managing Editor \\ University of California \\ Los Angeles 24, California
}

${ }^{*}$ During the absence of Herbert Busemann in 1952.

\section{ASSOCIATE EDITORS}

$\begin{array}{llll}\text { R. P. DILWORTH } & \text { P. R. HALMOS } & \text { B } \emptyset \text { RGE JESSEN } & \text { J. J. STOKER } \\ \text { HERBERT FEDERER } & \text { HEINZ HOPF } & \text { PAUL LÉVY } & \text { E. G. STRAUS } \\ \text { MARSHALL HALL } & \text { R. D. JAMES } & \text { GEORGE PÓLYA } & \text { KÖSAKU YOSIDA }\end{array}$

\section{SPONSORS}

UNIVERSITY OF BRITISH COLUMBIA

CALIFORNIA INSTITUTE OF TECHNOLOGY

U NIVERSITY OF CALIFORNIA, BERKELEY

UNIVERSITY OF CALIFORNIA, DAVIS

UNIVERSITY OF CALIFORNIA, LOS ANGELES

UNIVERSITY OF CALIFORNIA, SANTA BARBARA

OREGON STATE COLLEGE

UNIVERSITY OF OREGON

\author{
UNIVERSITY OF SOUTHERN CALIFORNIA \\ STANFORD UNIVERSITY \\ WASHINGTON STATE COLLEGE \\ UNIVERSITY OF WASHINGTON \\ AMERICAN MATHEMATICAL SOCIETY \\ NATIONAL BUREAU OF STANDARDS, \\ INSTITUTE FOR NUMERICAL ANALYSIS
}

Mathematical papers intended for publication in the Pacific Journal of Miathematics should be typewritten (double spaced), and the author should keep a complete copy. Manuscripts may be sent to any of the editors. All other communications to the editors should be addressed to the managing editor, E. F. Beckenbach, at the address given above.

Authors are entitled to receive 100 free reprints of their published papers and may obtain additional copies at cost.

The Pacific Journal of Mathematics is published quarterly, in March, June, September, and December, by the University of California, Berkeley 4, California. The price per volume (4 numbers) is $\$ 8.00$; single issues, $\$ 2.50$. Special price to individual faculty members of supporting institutions and to individual members of the American Mathematical Society: $\$ 4.00$ per volume; single issues, $\$ 1.25$.

Subscriptions, orders for back numbers, and changes of address should be sent to the publishers, University of California Press, Berkeley 4, California.

Printed at Ann Arbor, Michigan. Entered as second class matter at the Post Office, Berkeley, California.

\section{UNIVERSITY OF CALIFORNIA PRESS • BERKELEY AND LOS ANGELES}




\section{Pacific Journal of Mathematics}

\section{Vol. 2, No. $3 \quad$ March, 1952}

Lars V. Ahlfors, Remarks on the Neumann-Poincaré integral equation .... 271

Leonard P. Burton, Oscillation theorems for the solutions of linear, nonhomogeneous, second-order differential systems ............ 281

Paul Civin, Multiplicative closure and the Walsh functions . . . . . . . . . . . 291

James Michael Gardner Fell and Alfred Tarski, On algebras whose factor algebras are Boolean .................................. 297

Paul Joseph Kelly and Lowell J. Paige, Symmetric perpendicularity in Hilbert geometries .................................. 319

G. Kurepa, On a characteristic property of finite sets .............. 323

Joseph Lehner, A diophantine property of the Fuchsian groups ......... 327

Donald Alan Norton, Groups of orthogonal row-latin squares ........... 335

R. S. Phillips, On the generation of semigroups of linear operators ....... 343

G. Piranian, Uniformly accessible Jordan curves through large sets of relative harmonic measure zero ........................ 371

C. T. Rajagopal, Note on some Tauberian theorems of $O . S z \tilde{A} ; s z \ldots \ldots \ldots 377$

Halsey Lawrence Royden, Jr., A modification of the Neumann-Poincaré method for multiply connected regions .................... 385

George H. Seifert, A third order irregular boundary value problem and the associated series ...................................... 395

Herbert E. Vaughan, Well-ordered subsets and maximal members of ordered sets....

Hans F. Weinberger, An optimum problem in the Weinstein method for eigenvalues.

Shigeki Yano, Note on Fourier analysis. XXXI. Cesàro summability of Fourier series. 